UNIVERSIDADE DE SÃO PAULO

FFCLRP - DEPARTAMENTO DE BIOLOGIA

PROGRAMA DE PÓS-GRADUAÇÃO EM BIOLOGIA COMPARADA

ANA LUIZA VERA E SILVA

Revisão taxonômica do gênero Isocheles Stimpson, 1858 e Loxopagurus Forest, 1964

(Decapoda, Anomura, Diogenidae) por dados morfológicos e moleculares

RIBEIRÃO PRETO - SP 
ANA LUIZA VERA E SILVA

Revisão taxonômica do gênero Isocheles Stimpson, 1858 e Loxopagurus Forest, 1964

(Decapoda, Anomura, Diogenidae) por dados morfológicos e moleculares

\section{Versão Reduzida}

Dissertação apresentada à Faculdade de Filosofia, Ciências e

Letras de Ribeirão Preto da USP, como parte das exigências para a obtenção do título de Mestre em Ciências, Área: BIOLOGIA COMPARADA

Orientador: Prof. Dr. Fernando Luis Medina Mantelatto

RIBEIRÃO PRETO - SP 
Autorizo a reprodução e divulgação total ou parcial deste trabalho, por qualquer meio convencional ou eletrônico, para fins de estudo e pesquisa, desde que citada a fonte.

Vera-Silva, Ana Luiza

Revisão taxonômica do gênero Isocheles Stimpson, 1858 e Loxopagurus Forest, 1964 (Decapoda, Anomura, Diogenidae) por dados morfológicos e moleculares. Ribeirão Preto, 2018.

86 f. : il. ; $30 \mathrm{~cm}$

Dissertação de Mestrado, apresentada à Faculdade de Filosofia, Ciências e Letras de Ribeirão Preto/USP. Área de concentração: Biologia Comparada.

Orientador: Mantelatto, Fernando Luis Medina.

1. Paguroidea. 2. Chave de identificação. 3. América. 4. 16S. 5. COI. 6. H3. 
VERA-SILVA, A. L. Revisão taxonômica do gênero Isocheles, Stimpson 1858 e Loxopagurus Forest, 1964 (Decapoda, Anomura, Diogenidae) por dados morfológicos e moleculares. 2018. 86 f. Dissertação (Mestrado em Biologia Comparada) - Faculdade de Filosofia Ciências e Letras de Ribeirão Preto, Universidade de São Paulo, Ribeirão Preto, 2018.

Aprovado em:

Banca Examinadora

Prof. Dr.

Instituição:

Julgamento:

Prof. Dr.

Instituição:

Julgamento:

Prof. Dr.

Instituição:

Julgamento:

Prof. Dr.

Instituição:

Julgamento: 


\section{AGRADECIMENTOS}

Agradeço a meu orientador, Prof. Dr. Fernando Mantelatto, pela confiança em mim depositada para a realização deste trabalho e pelo incentivo e dedicação para cumprir este objetivo, desde os esforços em conseguir o material necessário, até a torcida pelo sucesso das PCRs. E também a todos os membros, ex-membros e agregados do Laboratório de Bioecologia e Sistemática de Crustáceos, amigos sempre dispostos a ajudar na rotina do laboratório e discutir os mais variados assuntos, científicos ou não.

Agradeço a CAPES (Ciências do Mar II Proc. 2005/2014 - 23038.004308/2014-14), pela concessão da bolsa de mestrado para a realização deste projeto.

Aos projetos que proporcionaram todo o apoio financeiro e logístico, direta ou indiretamente, para o desenvolvimento desta pesquisa junto ao LBSC, concedidos e/ou coordenados do Prof. Dr. Fernando Mantelatto: Fundação de Amparo à Pesquisa do Estado de São Paulo (FAPESP) - Procs. No 1998/07454-5 e 2002/08178- 9 (Projetos Individuais de Pesquisa), 2010/50188-8 (Projeto Temático Biota), 2009/54931-0 (Projeto Coleções Científicas); à Coordenação de Aperfeiçoamento de Pessoal de Nível Superior (CAPES) Procs. No 315/2009 (Projeto Cooperação Internacional) e 2005/2014 - 23038.004308/201414 (Ciências do Mar II); ao Conselho Nacional de Desenvolvimento Científico e Tecnológico (CNPq) - Procs. 472746/2004-9, 471794/2006-6, 473050/2007-2, 471011/2011-8 (Edital Universal, Auxilio Individual a Pesquisa), 491490/2004-6, 490122/2006-0, 490353/2007-0 (Projetos Cooperação Internacional); 301359/2007-5, 302748/2010-5 (Produtividade em Pesquisa).

Ao Departamento de Biologia e ao Programa de Pós-Graduação em Biologia Comparada da Faculdade de Filosofia, Ciências e Letras de Ribeirão Preto da Universidade de São Paulo (FFCLRP/USP), por todo apoio e suporte logístico. 
Muito obrigada aos curadores e responsáveis pelas coleções de onde recebemos empréstimos de animais ou tecidos, ou buscamos informações a respeito de material das espécies de interesse. Pela atenção e indispensável ajuda agradeço Adam R. Wall, do Natural History Museum of Los Angeles County; Lily Berniker e Estefania Rodriguez, do American Museum of Natural History; Darryl L. Felder, da University of Louisiana at Lafayette Zoological Collection; Rafael Lemaitre, do National Museum of Natural History, Smithsonian Institution; Ingo Wehrtmann, do Museo de Zoología da Universidad de Costa Rica; Manuel Ayón-Parente, da Universidad de Guadalajara; Laure Carbari, Paula Martin-Lefevre e Inmaculada Frutos, do Muséum National d'Histoire Naturelle; Adam Baldinger, do Museum of Comparative Zoology; Christina Piotrowski, da California Academy of Sciences; Karen van Dorp, do Naturalis Biodiversity Center; Paul Callomon, da Academy of Natural Sciences of Drexel University, Philadelphia; Irene Cardoso, do Museu Nacional do Rio de Janeiro; e Marcos Tavares, do Museu de Zoologia da USP, que também mediou empréstimos do Museum of Comparative Zoology e California Academy of Sciences. Pelo apoio aos empréstimos e análise de material em coleções do exterior agradeço imensamente Ana Francisca Tamburus Gomes, Raquel Buranelli e Tatiana Magalhães.

Agradeço a minha família e amigos pelo carinho, apoio e paciência sempre, seja nos períodos de calmaria ou tempestade. 


\section{RESUMO}

VERA-SILVA, A. L. Revisão taxonômica do gênero Isocheles Stimpson, 1858 e Loxopagurus Forest, 1964 (Decapoda, Anomura, Diogenidae) por dados morfológicos e moleculares. 2018. 86 f. Dissertação (Mestrado em Biologia Comparada) - Faculdade de Filosofia Ciências e Letras de Ribeirão Preto, Universidade de São Paulo, Ribeirão Preto, 2018.

Os gêneros Isocheles e Loxopagurus são endêmicos da América e ocorrem em águas tropicais e subtropicais. Isocheles é composto por cinco espécies, duas encontradas no Atlântico oeste (I. sawayai e I. wurdemanni) e três no Pacífico leste (I. pilosus, I. pacificus, e I. aequimanus). Loxopagurus é um gênero monotípico e ocorre apenas na costa sudeste da América do Sul. Estes dois gêneros são morfologicamente semelhantes, tendo como principal diferença a heteroquelia em Loxopagurus em contraste com a isoquelia de Isocheles. Há poucas informações na literatura sobre estes gêneros, e dúvidas quanto a seu status taxonômico foram recentemente ressuscitadas. Dessa forma este estudo visa elucidar as relações filogenéticas de Isocheles e Loxopagurus e avaliar a sua validade taxonômica. Para isso, foram realizadas análises moleculares utilizando os genes $16 \mathrm{~S}$ rRNA, COI e H3, e análises morfológicas, buscando caracteres que facilitem a identificação destas espécies, bem como a contextualização de ambos os gêneros dentro da Família. Os tipos de I. aequimanus e I. pilosus foram perdidos e erros de identificação entre as espécies de Isocheles são bem comuns. Assim, foram propostos caracteres que delimitam de maneira clara estas espécies, como ornamentação e formato dos quelípodos e o número de dentes presentes no segundo segmento da antena. A proposição de neótipos não foi necessária, uma vez que não há problemas taxonômicos entre estas espécies que não puderam ser resolvidos com base nas descrições originais e desenhos. Um dos lotes de I. wurdemanni do Museum of Comparative Zoology foi identificado pelo presente estudo como o holótipo da espécie. Isocheles e 
Loxopagurus se mostraram gêneros monofiléticos distintos, com base nos 3 marcadores utilizados, e grupos irmãos dentro de Diogenidae. Além disso, constatou-se que as espécies de Isocheles também formam grupos monofiléticos e uma nova espécie foi encontrada, divergindo tanto morfológica quanto molecularmente das demais. Assim, com base na morfologia, na topologia das árvores geradas e nas distâncias genéticas, conclui-se que, não somente os dois gêneros, mas também as espécies englobadas, são unidades taxonômicas distintas válidas.

Palavras-chave: Paguroidea. Chave de identificação. América. 16S. COI. H3. 


\begin{abstract}
VERA-SILVA, A. L. Taxonomic revision of the genus Isocheles Stimpson, 1858 and Loxopagurus Forest, 1964 (Decapoda, Anomura, Diogenidae) using morphological and molecular data. 2018. 86 f. Dissertação (Mestrado em Biologia Comparada) Faculdade de Filosofia Ciências e Letras de Ribeirão Preto, Universidade de São Paulo, Ribeirão Preto, 2018.

The genera Isocheles and Loxopagurus are endemic to America and occur in tropical and subtropical waters. There are five species of Isocheles, two of them are found in West Atlantic (I. sawayai and I. wurdemanni) and three are found in East Pacific (I. pilosus, I. pacificus and I. aequimanus). Loxopagurus is a monotypic genus and occurs only in southeast coast of South America. These two genera are morphologically similar, but the main difference between them is the heterochelia of Loxopagurus, while the chelipeds of Isocheles are similar in shape and size. There are few published information about these genera, and some doubts about their taxonomic status arised recently. Thus, this study aims to elucidate the phylogenetic relationship of Isocheles and Loxopagurus and evaluate their taxonomic validity. For that, molecular analyses were performed based on the genes $16 \mathrm{~S}$ rRNA, COI and $\mathrm{H} 3$, as well as morphological analysis, seeking characters that facilitate the identification of these species, and that contextualize both genera in the family. The type specimens of $I$. aequimanus and I. pilosus were lost and identification errors are common between Isocheles species. Therefore, characters that clearly delimit these species were stated, as the ornamentation and shape of the chelipeds and the number of teeth in the second segment of the antenna. There was no need to designate neotypes, once there are no taxonomic problems between the species that could not be solved based on the original descriptions and illustrations. Also, we identified that one of the lots of I. wurdemanni from the Museum of Comparative Zoology is the holotype of the species. The analysis based on the three
\end{abstract}


molecular markers showed that Isocheles and Loxopagurus are two different monophyletic genera, and sister taxa among Diogenidae. We also verified that each one of the species of Isocheles are monophyletic, and found a new species that differs both molecular and morphologically from the others. Thus, based on the morphology, on the tree topology and on genetic divergences, we concluded that not only the two genera, but also the species that they encompass, are valid taxonomic units.

Keywords: Paguroidea. Identification key. America. 16S. COI. H3. 
SUMÁRIO

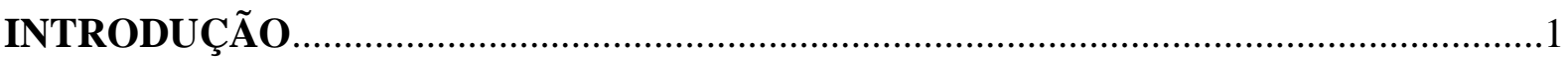

OBJETIVOS

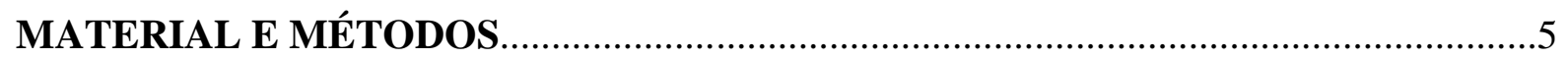

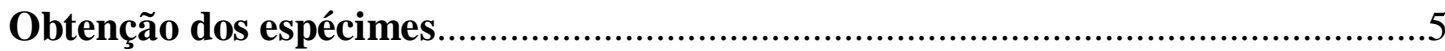

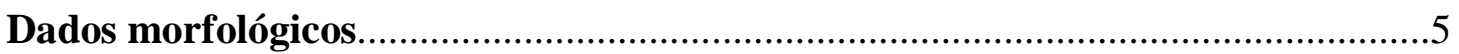

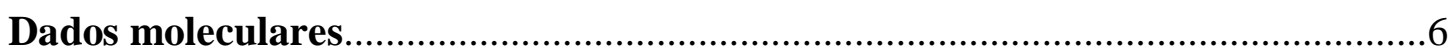

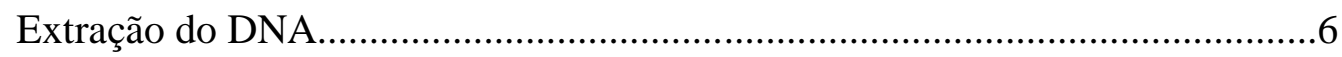

Escolha dos marcadores..................................................................

Amplificação do DNA..........................................................................

Purificação e sequenciamento.................................................................. 9

Edição das sequências.............................................................................

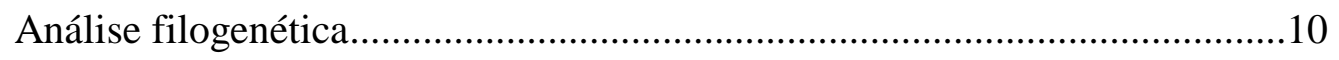

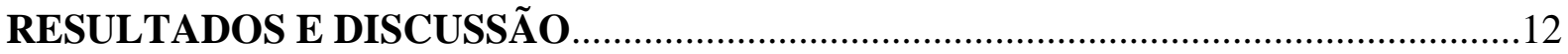

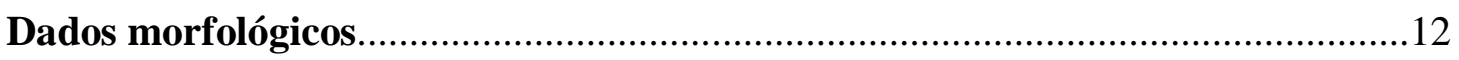

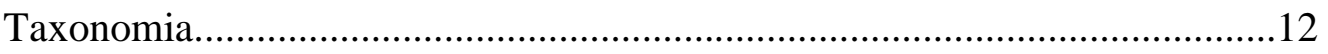

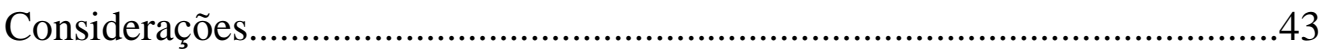

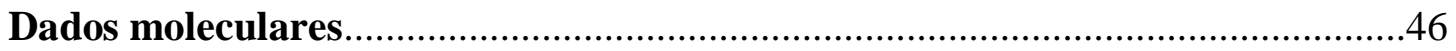

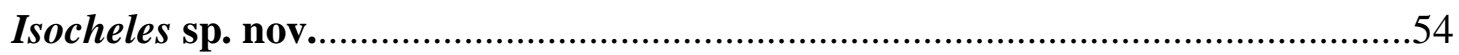

Chave de identificação para Isocheles.......................................................59

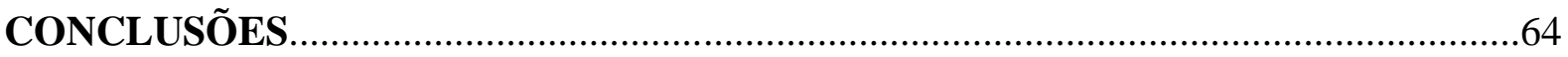

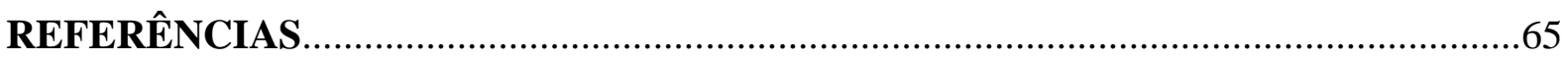




\section{INTRODUÇÃO}

Os gêneros Isocheles STIMPSON, 1858 e Loxopagurus FOREST, 1964 fazem parte da família Diogenidae, ermitões com tendência à assimetria favorecendo o lado esquerdo (MCLAUGHLIN, 1983).

O gênero Isocheles engloba cinco espécies: Isocheles pilosus (HOLMES, 1900), I. pacificus (BOUVIER, 1907), I. aequimanus (DANA, 1852), I. sawayai FOREST; DE SAINT LAURENT, 1968, e I. wurdemanni STIMPSON, 1859, restritas à América, sendo as três primeiras encontradas na costa oeste e as demais na costa leste. Estes ermitões são encontrados em águas rasas, em regiões tropicais e subtropicais do continente (FOREST; DE SAINT LAURENT, 1968).

Há grande semelhança interespecífica entre Isocheles: Isocheles pacificus já foi considerada uma variação de I. wurdemani (CHIRICHIGNO, 1970), e ambas são similares a I. aequimanus. Guzmán (2004) apresentou uma diagnose e propôs uma chave de identificação para I. aequimanus, além de confirmar a presença dessa espécie no Chile, antes considerada com distribuição desconhecida (FOREST; DE SAINT LAURENT, 1968). Forest e de Saint Laurent (1968) destacaram a semelhança existente entre I. sawayai e I. wurdemani, corroborada por Nucci e Melo (2015), e sugeriram caracteres para diferenciá-las. Há sobreposição de ocorrência destas duas espécies na Venezuela, porém I. sawayai ocorre também no Brasil, enquanto I. wurdemanni ocorre no Golfo do México e Colômbia (PROVENZANO, 1959; FOREST; DE SAINT LAURENT, 1968; SÁNCHEZ; CAMPOS, 1978; MELO, 1999; MANTELATTO et al., 2006; NUCCI; MELO, 2015).

Já Loxopagurus é monotípico, apresentando como única espécie Loxopagurus loxochelis (MOREIRA, 1901), e é endêmico da costa sudeste atlântica. No Brasil é encontrada do Rio de Janeiro ao Rio Grande do Sul, e também ocorre no Uruguai e Argentina (MELO, 1999; MANTELATTO et al., 2004). Esta espécie pode co-ocorrer com Isocheles 
sawayai em algumas áreas, mas aparentemente não há competição por recursos entre elas, uma vez que existem diferenças em sua distribuição espacial (STANSKI et al., 2016) e na ocupação de conchas (BIAGI et al., 2006; FANTUCCI et al., 2008; GALINDO et al., 2008; AYRES-PERES et al., 2012). Isocheles sawayai é encontrado em regiões costeiras, preferencialmente até $5 \mathrm{~m}$ de profundidade ou menos (FANTUCCI et al., 2009). Já Loxopagurus loxochelis é encontrado entre 12 e $30 \mathrm{~m}$ de profundidade (AYRES-PERES; MANTELATTO, 2008; FRANSOZO et al., 2008).

Loxopagurus loxochelis foi inicialmente descrito por Moreira (1901) como uma espécie de Pagurus Fabricius, 1775. Uma característica desse gênero é ter o flagelo da antena nu, no entanto, L. loxochelis apresenta cerdas longas nessa estrutura. Considerando esta diferença, Forest (1964) criou o gênero Loxopagurus, e realocou Pagurus loxochelis neste gênero. Além disso, análises morfológicas da carapaça, pedúnculos oculares e antenulares, e das peças bucais revelaram grande afinidade de Loxopagurus com Isocheles (FOREST, 1964). Loxopagurus é morfologicamente similar a Isocheles, sendo a relação entre os quelípodos a principal diferença entre estes gêneros, subiguais em tamanho e forma em Isocheles e bastante desiguais em Loxopagurus (FOREST, 1964; MELO, 1999).

Foi apontada por Moran (1984) a necessidade de estudos sobre o gênero Isocheles, e ainda hoje, apesar de estudos sobre ocupação de conchas e ecologia serem relativamente abundantes, são poucos os estudos sobre estes ermitões referentes a sistemática e taxonomia, não havendo trabalhos que comparem as cinco espécies do gênero. Em alguns casos, informações conflitantes dificultam a identificação das espécies de Isocheles e levantam dúvidas sobre o status taxonômico de Loxopagurus. Com base no gene mitocondrial $16 \mathrm{~S}$ rRNA, Mantelatto et al. (2006) mostraram a separação de Isocheles e Loxopagurus em dois clados monofiléticos. Contudo, Nucci e Melo (2015) afirmaram que mais estudos envolvendo 
estes dois gêneros são necessários, sob a possibilidade de Isocheles e Loxopagurus serem sinonimizados. 


\section{OBJETIVOS}

Considerando as semelhanças entre as espécies de Isocheles e Loxopagurus, este estudo visa elucidar as relações filogenéticas entre os dois gêneros, buscando suporte nas evidencias moleculares e morfológicas que permitam a distinção mais clara destes táxons e a avaliação de seu status taxonômico.

Adicionalmente, objetivou-se verificar o posicionamento destes dois gêneros na família e as relações entre as espécies de Isocheles. Considerando a ausência de trabalhos que comparem estas cinco espécies, buscaram-se caracteres que permitam a distinção de todas. 


\section{CONCLUSÕES}

As análises morfológicas e moleculares corroboraram a validade taxonômica de Isocheles e Loxopagurus como dois gêneros distintos, com base nas diferenças no escudo cefálico e nos quelípodos, na topologia das árvores e nas distâncias genéticas. Estes gêneros são grupos irmãos e, assim como as espécies englobadas por eles, são monofiléticos.

Embora dois dos tipos das cinco espécies estejam perdidos, não é necessária a designação de neótipos, uma vez que as dúvidas taxonômicas surgidas até o momento puderam ser sanadas pelas descrições e desenhos disponíveis destes espécimes. Além disso, as análises moleculares apontaram para a existência de uma sexta espécie de Isocheles, que foi corroborada pela análise morfológica.

Apesar de bastante semelhantes, as espécies de Isocheles são distinguíveis pela forma do sulco pós-frontal transverso, que apresenta vértices arredondados em I. aequimanus, I. pacificus, I. pilosus e Isocheles sp. nov., enquanto em I. sawayai e I. wurdemanni os vértices são mais marcados; pela ornamentação dos quelípodos, com pelo menos 6 espinhos na extremidade distal da face dorsolateral do mero em I. aequimanus, ausentes nas demais espécies; quela e face dorsolateral do carpo com predominância de espinhos pontiagudos em I. pilosus e I. sawayai; tubérculos com fileiras laterais e dorsais de espinhos ou dentes, menos proeminentes que nas outras espécies, em I. pacificus e I. wurdemanni; fileiras de espinhos apenas nas laterais da quela em I. aequimanus; e sem espinhos na quela em Isocheles sp. nov.; abaulamento abrupto entre o centro e o ápice da palma dos quelípodos de I. pilosus, enquanto nas demais espécies a lateral da quela é apenas levemente convexa e em Isocheles sp. nov. há abaulamento abrupto, porém na base da palma; e pelo número de dentes no segundo segmento da antena, entre 6 e 9 em I. aequimanus e 4 ou menos nas demais espécies. 


\section{REFERÊNCIAS}

ABELE, L. G.; KIM, W. 1986. An Illustrated Guide to the Marine Decapod Crustaceans of Florida. Tallahassee, Florida: Florida State University. p. 225.

ALCOCK, A. 1905. Catalogue of the Indian decapod Crustacea in the collection of the Indian Museum: Part II. Anomura. Fasciculus I. Pagurides. Calcutta: Trustees of the Indian Museum, xi. p. 193.

AYÓN-PARENTE, M.; HENDRICKX, M. E. 2010. Species richness and distribution of hermit crabs of the family Diogenidae (Crustacea: Decapoda: Anomura) in the eastern Pacific. Nauplius, 18(1): 1-12.

AYRES-PERES, L.; MANTELATTO, F. L. 2008. Patterns of distribution of the hermit crab Loxopagurus loxochelis (Moreira, 1901) (Decapoda, Diogenidae) in two coastal areas of southern Brazil. Revista de Biología Marina y Oceanografía, 43(2): 399-411.

AYRES-PERES, L.; QUADROS, A. F.; MANTELATTO, F. L. 2012. Comparative analysis of shell occupation by two southern populations of the hermit Loxopagurus loxochelis (Decapoda, Diogenidae). Brazilian Journal of Oceanography, 60(3): 299-310.

BALDINGER, A. J. 1999. The Crustacean Collection at the Museum of Comparative Zoology, Harvard University. In: SCHRAM, F. R.; VAUPEL-KLEIN, J. C. (Ed.). Proceedings of the Fourth International Crustacean Congress. Amsterdam, the Netherlands: 1998. Vol. 1, p. 45-59.

BIAGI, R.; MEIRELES, A. L.; SCELZO, M. A.; MANTELATTO, F. L. 2006. Comparative study of shell choice by the southern endemic hermit crab Loxopagurus loxochelis from Brazil and Argentina. Revista Chilena de Historia Natural, 79: 481-487.

BORDWELL, C. 1991. Delay and Wreck of the Peacock: An Episode in the Wilkes Expedition. Oregon Historical Quarterly, 92(2): 119-198. 
BOSCHI, E. E. 1979. Geographic distribution of Argentinian marine decapod crustaceans. Bulletin of the Biological Society of Washington, 3: 134-143.

BOUVIER, E. L. 1907. Crustacés Décapodes Nouveaux Recueillis à Païta (Pérou) par M. le Dr Rivet. Extrait du Bulletin du Muséum national d'Histoire naturelle, 2: 113116.

BRACKEN-GRISSOM, H. D.; CANNON, M. E.; CABEZAS, P.; FELDMANN, R. M.; SCHWEITZER, C. E.; AHYONG, S. T.; FELDER, D. L.; LEMAITRE, R.; CRADALL, K. A. 2013. A comprehensive and integrative reconstruction of the evolutionary history for Anomura (Crustacea: Decapoda). BMC Evolutionary Biology, 13: 128.

CARVALHO, F. L.; PILEGGI, L. G.; MANTELATTO, F. L. 2013. Molecular data raise the possibility of cryptic species in the Brazilian endemic prawn Macrobrachium potiuna (Decapoda, Palaemonidae). Latin American Journal of Aquatic Research, 41(4): 707-717.

CHIRICHIGNO, N. 1970. Lista de crustáceos del Perú (Decapoda y Stomatopoda) con datos de su distribución geográfica. Informe Instituto del Mar del Perú, 35: 1-95.

COELHO, P. A.; RAMOS, M. A. 1972. A constituição e a distribuição da fauna de decápodos do litoral leste da América do Sul entre as Latitudes de $5^{\circ} \mathrm{N}$ e $39^{\circ} \mathrm{S}$. Trabalhos Oceanográficos da Universidade Federal de Pernambuco, 12: 133-236.

COELHO, P. A.; RAMOS-PORTO, M. 1987. Sinopse dos crustáceos decápodes brasileiros (famílias Callianassidae, Callianeidae, Upogebiidae, Parapaguridae, Paguridae, Diogenidae). Trabalhos Oceanográficos, 19: 27-53.

COELHO, P. A.; SANTOS, M. F. A. 1980. Zoogeografia marinha do Brasil. I. Considerações gerais sobre o método e aplicação a um grupo de crustáceos (Paguros: Crustacea, 
Decapoda, superfamílias Paguroidea e Coenobitoidea). Boletim do Instituto de Oceanografia de São Paulo, 29 (2): 139-144.

COLGAN, D. J.; MCLAUCHLAN, A.; WILSON, G. D. F.; LIVINGSTONE, S. P.; EDGECOMBE, G. D.; MACARANAS, J.; CASSIS, G.; GRAY, M. R. 1998. Histone H3 and U2 snRNA DNA sequences and arthropod molecular evolution. Australian Journal of Zoology, 46: 419-437.

COSTA, H. R. 1962. Ocorrência do gênero Isocheles Stimpson, na costa brasileira. Centro de Estudos Zoológicos, 17: 1-3.

DANA, J. D. 1852. Crustacea. Part I. In: SHERMAN, C. (Ed.). United States Exploring Expedition during the years 1838, 1839, 1840, 1841, 1842, under the command of Charles Wilkes, U.S.N. Philadelphia. Vol. 13, p. 1-685.

DANA, J. D. 1853. On the classification and geographical distribution of Crustacea: from the report on Crustacea of the United States Exploring Expedition under Captain Charles Wilkes, U.S.N., during the years 1838-1842. Philadelphia: C. Sherman. p. 1395-1592.

DARRIBA, D.; TABOADA, G. L.; DOALLO, R.; POSADA, D. 2012. jModelTest 2: more models, new heuristics and parallel computing. Nature Methods, 9 (8): 772.

ESTOUP, A.; LAGIARDÈR C. R.; PERROT, E.; CHOURROUT, D. 1996. Rapid one-tube DNA extraction for reliable PCR detection of fish polypmorphic markers and transgenes. Molecular Marine Biology and Biotechnology, 5 (4): 295-298.

FANTUCCI, M. Z.; BIAGI, R.; MANTELATTO, F. L. 2008. Shell occupation by the endemic western Atlantic hermit crab Isocheles sawayai (Diogenidae) from Caraguatatuba, Brazil. Brazilian Journal of Biology, 68(4): 859-867.

FANTUCCI, M. Z.; BIAGI, R.; MEIRELES, A. L.; MANTELATTO, F. L. 2009. Influence of biological and environmental factors on the spatial and temporal distribution of the 
hermit crab Isocheles sawayai Forest e Saint-Laurent, 1968 (Anomura, Diogenidae). Nauplius, 17(1): 37-47.

FAUSTO-FILHO, J. 1970. Quarta Contribuição ao Inventário dos Crustáceos Decápodes Marinhos do Nordeste Brasileiro. Arquivos de Ciências do Mar, 10(1): 55-60.

FOREST, J. 1964. Sur un nouveau genre de Diogenidae (Crustacea Paguridea) de l'Atlantique sud-américain, Loxopagurus gen. nov., établi pour Pagurus loxochelis Moreira. Zoologische Mededelingen, 39: 279-296.

FOREST, J.; DE SAINT LAURENT, M. 1968. Campagne de la Calypso au large des côtes atlantiques de l'Amérique du Sud (1961-1962). 6. Crustacés decápodes: pagurides. Annales de l'Institut océanographique, 45: 47-169.

FRANCISCO, A. K.; GALETTI JUNIOR, P. M. 2005. Genetic distance between broodstocks of the marine shrimp Litopenaeus vannamei (Decapoda, Penaeidae) by mtDNA analyses. Genetics and Molecular Biology, 28 (2): 258-261.

FRANSOZO, A.; BERTINI, G.; BRAGA, A. C. A.; NEGREIROS-FRANSOZO, M. L. 2008. Ecological aspects of hermit crabs (Crustacea, Anomura, Paguroidea) off the northern coast of São Paulo state Brazil. Aquatic Ecology, 42(3): 437-448.

GALINDO, L. A.; BOLANOS, J. A.; MANTELATTO, F. L. 2008. Shell utilization pattern by the hermit crab Isocheles sawayai Forest and Saint Laurent, 1968 (Anomura, Diogenidae) from Margarita Island, Caribbean Sea, Venezuela. Gulf and Caribbean Research, 20: 49-57.

GUZMÁN, G. 2004. Isocheles aequimanus (Dana, 1852) (Decapoda, Anomura, Paguroidea): revalidación para la carcinofauna chilena. Investigaciones Marinas, 32 (2): 129-132.

HAIG, J. 1955. The Crustacea Anomura of Chile: Reports of the Lund University Chile Expedition 1948-1949. Lunds Universitets Arsskrift, 51(12): 1-68. 
HAIG, J.; HOPKINS, T. S.; SCANLAND, T. B. 1970. The shallow water anomuran crab fauna of southwestern Baja California, Mexico. Transactions of the San Diego Society of Natural History, 16(2): 12-31.

HAIG, J.; WICKSTEN, M. K. 1975. First records and range extensions of the crabs in California waters. Bulletin of the Southern California Academy of Sciences, 74(3): 100-104.

HALL, T. A. 2005. BioEdit 7.0.5. North Carolina State University, Department of Microbiology. [acesso 9 fev. 2018]. Disponível em: <http://www.mbio.ncsu.edu/BioEdit/bioedit.html>.

HARRISON, J. S. 2004. Evolution, biogeography, and the utility of mitochondrial 16s and COI genes in phylogenetic analysis of the crab genus Austinixa (Decapoda: Pinnotheridae). Molecular Phylogenetics and Evolution, 30: 743-754.

HARVARD UNIVERSITY. 1873. Annual report of the trustees of the Museum of Comparative Zoology at Harvard College, in Cambridge: together with the report of the director for 1872. Boston: Wright; Potter, State Printers, p. 4-36.

HEBLING， N. J.; MANTELATTO, F. L. M.; NEGREIROS-FRANSOZO, M. L.; FRANSOZO, A. 1994. Levantamento e distribuição de braquiúros e anomuros (Crustacea, Decapoda) dos sedimentos sublitorais da região de Ilha Anchieta, Ubatuba (SP). Boletim do Instituto de Pesca, 21: 1-9.

HENDRICKX, M. E.; AYÓN-PARENTE, M.; PICO, E. F.; LÓPEZ, V. G. V. 2008. Hermit crabs (Crustacea: Paguroidea) in the biological collection of CICIMAR, Instituto Politecnico Nacional, La Paz, Baja California Sur, Mexico. Contributions to the study of East Pacific Crustaceans, 5(1): 17-21. 
HENDRICKX, M. E.; HARVEY, A. W. 1999. Checklist of Anomuran Crabs (Crustacea: Decapoda) from the Eastern Tropical Pacific. Belgian Journal of Zoology, 129(2): 363-389.

HOLMES, S. J. 1900. Synopsis of California Stalk-eyed Crustacea. Occasional Papers of the California Academy of Sciences, 7: 1-262.

ICZN (International Commission on Zoological Nomenclature). 1999. International Code of Zoological Nomenclature. 4th ed. Londres: The international Trust for Zoological Nomenclature. p. 306.

KAMEYA, A.; MOSCOSO, V.; LLEELliSH, M. 1998. Los Crustáceos Decápodos y Estomatópodos del Perú. Informe del Instituto del Mar del Perú, 136: 80-109.

KATOH, K.; STANDLEY, D. M. 2013. MAFFT multiple sequence alignment software version 7: improvements in performance and usability. Molecular Biology and Evolution, 30(4): 772-780.

KRELL, F. T.; MARSHALL, S. A. 2017. New Species Described From Photographs: Yes? No? Sometimes? A Fierce Debate and a New Declaration of the ICZN. Insect Systematics and Diversity, 1(1): 3-19.

LIU, M. Y.; CAI, Y. X.; TZENG, C. S. 2007. Molecular systematics of the freshwater prawn genus Macrobrachium Bate, 1868 (Crustacea: Decapoda: Palaemonidae) inferred from mtDNA sequences, with emphasis on East Asian species. Zoological Studies, 46(3): 272-289.

MANNING, R. B. 1993. The Scientific Contributions of William Stimpson, an early American Naturalist and Taxonomist. Crustacean Issues, 8: 109-117.

MANTELATTO, F. L.; MARTINELLI, J. M.; FRANSOZO, A. 2004. Temporal-spatial distribution of the hermit crab Loxopagurus loxochelis (Decapoda: Diogenidae) from Ubatuba Bay, São Paulo State, Brazil. Revista de Biología Tropical, 52(1): 47-55. 
MANTELATTO, F. L.; ROBLES, R.; FELDER, D. L. 2007. Molecular phylogeny of the Western Atlantic species of the genus Portunus (Crustacea, Brachyura, Portunidae). Zoological Journal of the Linnean Society of London, 150(1): 211-220.

MANTELATTO, F. L.; ROBLES, R.; BIAGI, R.; FELDER, D. L. 2006. Molecular analysis of the taxonomic and distributional status for the hermit crab genera Loxopagurus Forest, 1964 and Isocheles Stimpson, 1858 (Decapoda, Anomura, Diogenidae). Zoosystema, 28(2): 495-506.

MCLAUGHLIN, P. A. 1983. Hermit crabs: Are they really polyphyletic? Journal of Crustacean Biology, 3(4): 608-621.

MCLAUGHLIN, P. A.; KOMAI, T.; LEMAITRE, R.; RAHAYU, D. L. 2010. Annotated checklist of anomuran decapod crustaceans of the world (exclusive of the Kiwaoidea and families Chirostylidae and Galatheidae of the Galatheoidea): Part I - Lithodoidea, Lomisoidea and Paguroidea. The Raffles Bulletin of Zoology 2010, Supplement No. 23: 5-107.

MELO, G. A. S. 1999. Manual de identificação dos Crustacea Decapoda do litoral brasileiro: Anomura, Thalassinidea, Palinuridea, Astacidea. São Paulo: Plêiade. p. 551.

MENDEZ, O. V.; PEREZ, A. L.; GUERRERO, B. M.; PEREZ, V. A.; CHAVEZ, E. R. 2017. A new record of Harlequin Shrimp (Malacostraca: Decapoda: Palaemonidae: Hymenocera picta Dana, 1852) in the southern Mexican Pacific Reefs. Journal of Threatened Taxa, 9(8): 10571-10576.

MORAN, D. A. 1984. Additions to the known anomuran fauna of El Salvador, Central America (Crustacea: Decapoda). Journal of Crustacean Biology, 4(1): 72-84.

MOREIRA, C. 1901. Contribuições para o conhecimento da fauna brasileira. Crustaceos do Brasil. Archivos do Museu Nacacional Rio de Janeiro, 11: 1-151. 
MOREIRA, C. 1906. Campanhas de pesca do "Annie". Crustáceos. Archivos do Museu Nacional do Rio de Janeiro, 13: 1-25.

MOSCOSO, V. 2012. Catálogo de Crustáceos Decápodos y Estomatópodos del Perú. Boletín del Instituto del Mar del Perú, 27(1-2): 1-209.

NEGREIROS-FRANSOZO, M. L.; FRANSOZO, A.; MANTELATTO, F. L.; PINHEIRO, M. A. A.; SANTOS, S. 1997. Anomuran species (Crustacea, Decapoda) and their ecological distribution at Fortaleza Bay sublittoral, Ubatuba, São Paulo, Brazil. Iheringia, Série Zoologia 83: 187-194.

NEGREIROS-FRANSOZO, M. L.; HEBLING, N. J. 1983. Desenvolvimento pósembrionário de Isocheles sawayai Forest e Saint Laurent, 1967 (Decapoda, Diogenidae), em Laboratório. Papéis Avulsos de Zoologia, 35(4): 41-53.

NEGRI, M. P.; LEMAITRE, R.; MANTELATTO, F. L. 2014. Molecular and morphological resurrection of Clibanarius symmetricus (Randall, 1840), a cryptic species hiding under the name for the "thinstripe" hermit crab C. vittatus (Bosc, 1802) (Decapoda: Anomura: Diogenidae). Journal of Crustacean Biology, 34(6): 848-861.

NEGRI, M.; PILEGGI, L. G.; MANTELATTO, F. L. 2012. Molecular barcode and morphological analyses reveal the taxonomic and biogeographical status of the striped-legged hermit crab species Clibanarius sclopetarius (Herbst, 1796) and Clibanarius vittatus (Bosc, 1802) (Decapoda: Diogenidae). Invertebrate Systematics, 26: 561-571.

NOEVER, C.; GLENNER, H. 2017. The origin of king crabs: hermit crab ancestry under the magnifying glass. Zoological Journal of the Linnean Society, 182(2): 300-318.

NUCCI, P. R.; MELO, G. A. S. 2000. Range extensions for eight species of western atlantic hermit crabs (Crustacea, Paguroidea). Nauplius, 8(1): 141-147. 
NUCCI, P. R.; MELO, G. A. S. 2015. Hermit crabs from Brazil: Family Diogenidae (Crustacea: Decapoda: Paguroidea), except Paguristes. Zootaxa, 3947 (3): 327-346.

ORTMANN, A. E. 1892. Die Decapoden-Krebse des Strassburger Museums, mit besonderer Berücksichtigung der von Herrn Dr. Döderlein bei Japan und bei den Liu-Kiu-Inseln gesammelten und zur Zeit im Strassburger Museum auf bewahrten Formen. IV Theil. Die Abtheilungen Galatheidea und Paguridea. Zoologischer Jahresbericht, 6: 241325.

PORTER, C. E. 1935. Catalogo de los paguridos de Chile. Revista Chilena de Historia Natural, 39: 134-137.

POUPIN, J. 1996. Crustacea Decapoda of French Polynesia (Astacidea, Palinuridea, Anomura, Brachyura. Attoll Research Bulletin, 442: 1-114.

PROVENZANO, A. J. Jr. 1959. The shallow-water hermit crabs of Florida. Bulletin of Marine Science of the Gulf and Caribbean, 9(4): 349-420.

RATHBUN, M. J. 1910. The stalk-eyed Crustacea of Peru and the adjacent coast. Proceedings of United States National Museum, 38: 531-620.

RICKETTS, E. F.; CALVIN, J.; HEDGPETH, J. W.; PHILliPS, D. W. 1985. Between Pacific tides. 5th ed. Stanford, California: Stanford University Press. p. 652.

RIEGER, P. J. 1997. Os “Ermitões” (Crustacea, Decapoda, Parapaguridae, Diogenidae e Paguridae) do Litoral do Brasil. Nauplius, 5(2): 99-124.

RONQUIST, F.; TESLENKO, M.; VAN DER MARK, P.; AYRES, D.; DARLING, A.; HÖHNA, S.; LARGET, B.; LUI, L.; SUCHARD, M. A.; HUELSENBECK, J. P. 2012. MrBayes 3.2: efficient Bayesian phylogenetic inference and model choice across a large model space. Systematic Biology, 61: 539-542. 
SÁNCHEZ, H. M.; CAMPOS, N. H. 1978. Los cangrejos ermitaños (Crustacea, Anomura, Paguridae) de la costa norte colombiana. Anales del Instituo de Investigaciones Marinas de Punta Betín, 10: 15-62.

SCELZO, M. A.; BOSCHI, E. E. 1973. Aportes al conocimiento de la distribuicion geografica de los crustaceos decapodos Anomura del Atlantico Sudoccidental, frente a las costas Argentinas. Trabajo V Congreso Latinoamericano de Zoologia, 1: 204216.

SCHMITT, W. L. 1921. The Marine Decapod Crustacea of California with Special Reference to the Decapod Crustacea Collected by the United States Bureau of Fisheries Steamer "Albatross" in Connection with the Biological Survey of San Francisco Bay during the Years 1912-1913. Berkeley, California: University of California Publications in Zoologi. Vol. 23, p. 470.

SCHUBART, C. D.; NEIGEL, J. E.; FELDER, D. L. 2000. Use of the mitochondrial 16S rRNA gene for phylogenetic and population studies of Crustacea. Crustacean Issues, 12(1): 817-830.

SMITHSONIAN LIBRARIES. [sem data]. Follow The U. S. Exploring Expedition. [acesso 9 fev. 2018]. Disponível em: <http://www.sil.si.edu/DigitalCollections/usexex/navigation/Crew/crew_display_by_s hip.cfm?ship=Vincennes $>$.

SNYDER-CONN, E. 1980. Arthropoda: Crustacea. Paguroidea and Coenobitoidea (Hermit Crabs). In: Brusca, R. C. (Ed.). Common Intertidal Invertebrates of the Gulf of California. 2nd ed. Arizona: The University of Arizona Press Tucson. p. 275-282.

STAMATAKIS, A. 2006. RAxML-VI-HPC: maximum likelihood-based phylogenetic analyses with thousands of taxa and mixed models. Bioinformatics, 22 (21): 26882690. 
STANSKI, G.; MANTELATTO, F. L.; CASTILHO, A. L. 2016. Habitat heterogeneity in the assemblages and shell use by the most abundant hermit crabs (Anomura: Diogenidae and Paguridae): does the occupied shell species differ according to gender and species? Nauplius, 24: e2016004.

STIMPSON, W. 1858. Crustacea Cancroidea et Corystoidea. Prodromus descriptionis animalium evertebratorum, quae in Expeditione ad Oceanum Pacificum Septentrionalem a Republica Federata missa, Cadwaladaro Ringgold et Johanne Rodgers ducibus, observavit et descripsit, Pars VII. Crustacea Anomura. Proceedings of the Academy of Natural Sciences of Philada, 10: 225-252.

STIMPSON, W. 1859. Notes on North American Crustacea. Annals Lyceum of Natural History, 7: 49-93.

STRASSER, K. M.; PRINCE, W. W. 1999. An annotated checklist and key to hermit crabs of Tampa Bay, Florida, and surrounding waters. Gulf Research Reports, 11(1): 33-50.

TAMURA, K.; PETERSON, D.; PETERSON, N.; STECHER, G.; NEI, M.; KUMAR, S. 2011. MEGA5: Molecular evolutionary genetics analysis using maximum likelihood, evolutionary distance and maximum parsimony methods. Molecular Biology and Evolution, 28(10): 2731-2739.

TIMM, L.; BRACKEN-GRISSOM, H. D. 2015. The forest for the trees: evaluating molecular phylogenies with an emphasis on higher-level Decapoda. Journal of Crustacean Biology, 35(5): 577-592.

TOON, A.; FINLEY, M.; STAPLES, J.; CRANDALL, K. A. 2009. Decapod Phylogenetics and Molecular Evolution. Crustacean Issues, 18: 15-29.

WALKER, S. E. 1989. Crabs as Taphonomic Agents. Palaios, 4(5): 439-452. 
WALSH, P. S.; METZGER, D. A.; HIGUCHI, R. 2013. Chelex 100 as a Medium for Simple Extraction of DNA for PCR-Based Typing from Forensic Material. BioTechniques, 54(3): 134-139.

WANG, Q.; WANG, X. 2012. Comparison of methods for DNA extraction from a single chironomid for PCR analysis. Pakistan Journal of Zoology, 44(2): 421-426.

WASS, M. L. 1955. The decapod crustaceans of Alligator Harbor and adjacent inshore areas of Northwestern Florida. Quarterly journal of the Florida Academy of Sciences, 18(3): $148-152$.

WESTERVELT, C. A. JR. 1967. The Littoral Anomuran Decapod Crustacean Fauna of the Punta Peñasco-Bahía la Cholla Area in Sonora, Mexico. Ph. D. Thesis, University of Arizona. p. 143.

WICKSTEN, M. K. 1979. Digging by the hermit crab Isocheles pilosus (Holmes). Crustaceana Supplement, 5: 100.

WICKSTEN, M. K. 1988. Antennary cast-net feeding in California hermit crabs (Decapoda, Paguridea). Crustaceana, 54(3): 321-322.

WICKSTEN, M. K. 2008. Decapod Crustacea of the Californian and Oregonian zoogeographic provinces. Scripps Institution of Oceanography Library Paper, 26: $1-413$.

WICKSTEN, M. K. 2012. Decapod Crustacea of the Californian and Oregonian Zoogeographic Provinces. Zootaxa, 3371, 307 p.

WILKES, C. 1844. Narrative of the United States Exploring Expedition during the years 1838, 1839, 1841, 1842. Philadelphia: C. Sherman. Vol. 1, p. 318. 